\title{
Multidimensional Nanoparticle Characterization through Ion Mobility-Mass Spectrometry
}

Chenxi Li, ${ }^{1,3}$ Amani L. Lee, ${ }^{2}$ Xiaoshuang Chen, ${ }^{1}$ William C. K. Pomerantz, ${ }^{2}$ Christy L. Haynes, ${ }^{2}$ Christopher J. Hogan Jr. ${ }^{1 *}$

${ }^{1}$ Department of Mechanical Engineering, University of Minnesota, Minneapolis, MN 55455, USA

${ }^{2}$ Department of Chemistry, University of Minnesota, Minneapolis, MN 55455, USA

${ }^{3}$ Current Address: Laboratory for Physical Chemistry, ETH Zurich, Zurich 8093, Switzerland

\section{Supporting Information}

\section{Corresponding Author}

*To whom correspondence should be addressed: hogan108@umn.edu 


\section{Information Available}

A TEM image of the UMNs

- Schematic diagram of the IM-MS system

- The relation between applied DMA/APM voltages and particle diameter/mass

- The IM-MS system transfer function

○ Size-mass spectra of solid silica particles

- TEM images of size selected UMN particles

- Replicate measurements of UMNs

○ $\quad \mathrm{N}_{2}$ physisorption measurements

○ Mass correction for a water monolayer

○ Comparison of $\frac{\boldsymbol{d n}}{\boldsymbol{d} \boldsymbol{d}_{\boldsymbol{p}}}$ between IM-MS, TEM, and DLS 


\section{A TEM image of the synthesized UMNs}

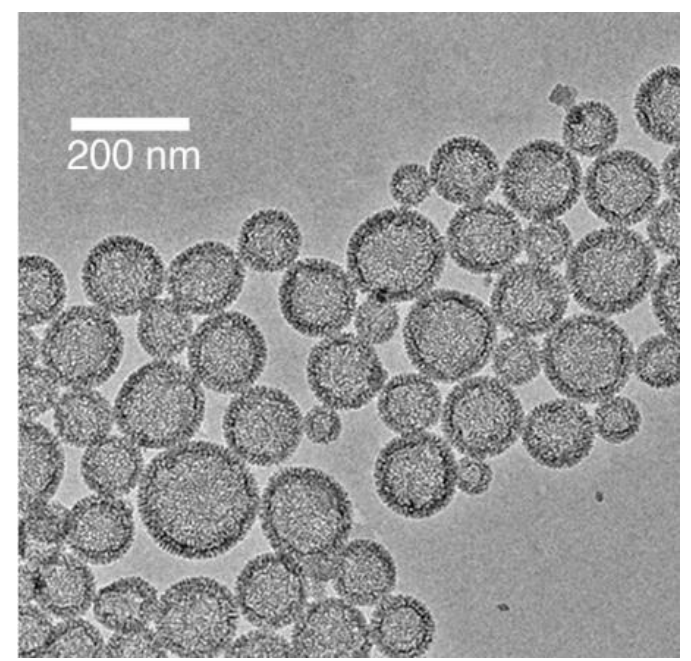

Figure S1. A TEM image of the UMNs. The sample was prepared by drop-cast on a TEM grid. 


\section{A detailed experimental schematic}

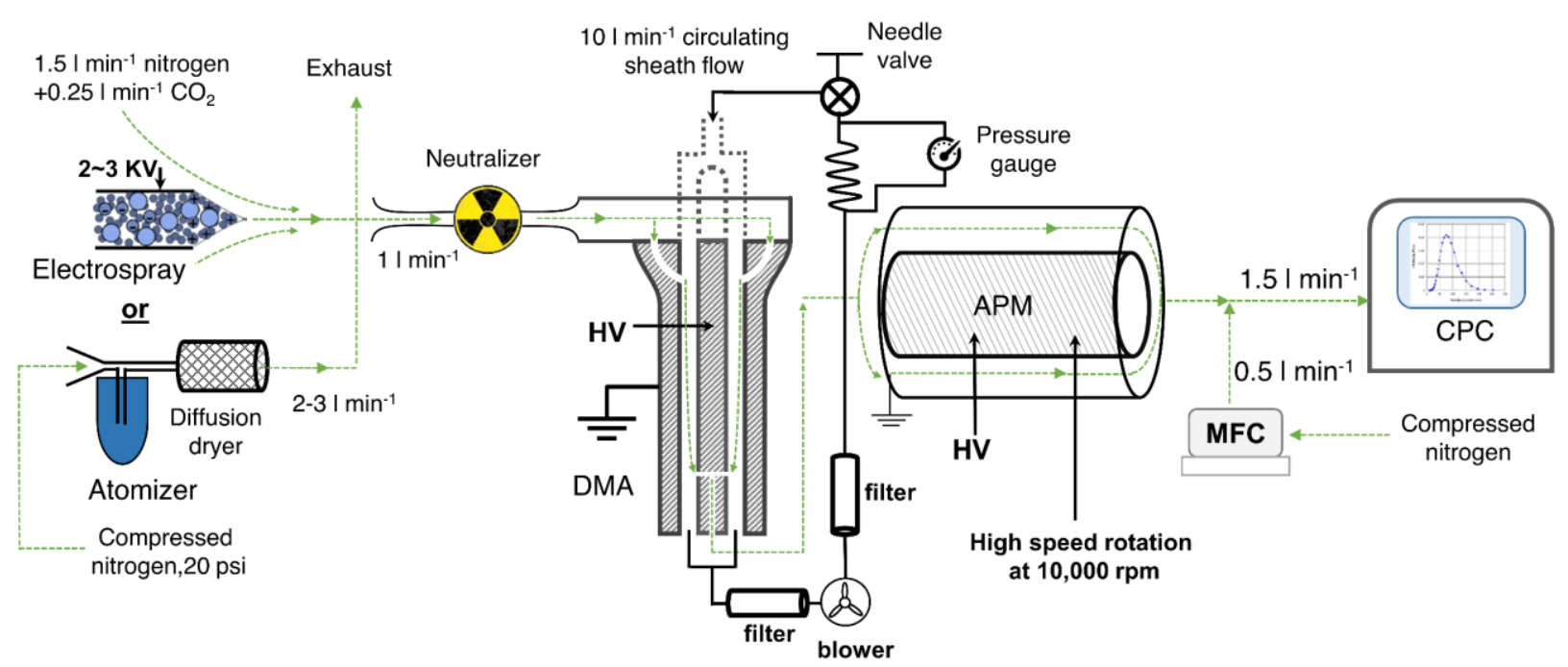

Figure S2. A schematic diagram with flow rates and instrument settings. The green dashed lines indicate the aerosol flow.

Figure S2 shows a schematic diagram of the IM-MS system with relevant flow rates and instrument settings for the DMA and APM. We utilized a commercial charge reduction electrospray apparatus (Model 3480, TSI Inc.) to aerosolize UMNs using a $\mathrm{N}_{2^{-}}$ $\mathrm{CO}_{2}$ gas mixture (total flowrate of $1.751 \mathrm{~min}^{-1}$ ) as carrier gas. UMN suspensions were electrosprayed without the addition of any solutes to enhance solution electrical conductivity, and using a 360- $\mu$ m-outer diameter 75- $\mu$ m-inner diameter silica capillary (Polymicro technologies, Phoenix, AZ). Cone-jet mode operation of the electrospray was confirmed by visual inspection of the electrospray tip using a magnifying lens. $\mathrm{CO}_{2}(0.25$ $1 \mathrm{~min}^{-1}$ ) was added to the carrier gas to avoid electrical discharge at the electrospray tip. For polystyrene latex particle standards, we used a home-built pneumatic atomizer to aerosolize the particles instead of electrospray, since the electrospray capillaries tend to clog after 1-2 hours of operation either by the solvent additives in the suspensions or by the deposition of the polystyrene latex nanoparticles themselves. For pneumatic 
atomization, 2-3 $1 \mathrm{~min}^{-1} \mathrm{~N}_{2}$ was used as carrier gas. A silica based diffusion drier was used downstream the atomizer to remove water vapour from the flow.

Regardless of the aerosolization method employed, $11 \mathrm{~min}^{-1}$ of particle laden flow was drawn into an aerosol "neutralizer" (Aerosol Neutralizer 3077, TSI Inc.) containing a ${ }^{85} \mathrm{Kr}$ radioactive source. ${ }^{85} \mathrm{Kr}$ emits $\beta$ particles, which ionize trace impurities in the carrier gas, creating a high concentration of charge carriers. The aerosolized particles interacted with these charge carriers by diffusion and the particle charge is reduced to a known charge state. ${ }^{1}$ Following charge conditioning, the particles entered a custom-built differential mobility analyzer with the same dimensions as the TSI DMA model $3081,^{2}$ operated with a closed-loop filtered sheath flow of $101 \mathrm{~min}^{-1}$. Particles of a specific ion mobility, determined by the balance of electrical force and drag force, traversed from DMA inlet to outlet. Particles transmitted through the DMA then entered the APM, where a centrifugal force was exerted on the particles as both the APM inner and outer cylinders rotated at a speed of 10,000 rotations per minute. Meanwhile, a voltage was applied between the inner and outer cylinders of the APM. Only particles with balanced electrical and centrifugal forces passed through the APM and reached the condensation particle counter (CPC, Model 3776, TSI Inc.). ${ }^{3}$ The CPC was operated with a flowrate of 1.51 $\min ^{-1}$; particle-free, $0.51 \mathrm{~min}^{-1} \mathrm{~N}_{2}$ was mixed with the aerosol flow exiting the APM in order to meet this flow requirement.

To obtain the 2D size-mass spectra of the particles, the DMA and APM voltages need to be stepped systematically to cover the desired particle size/mass range. To do so, we first set the DMA to a fixed voltage and stepped the APM applied voltage, with each step increasing the applied voltage by a factor of 1.08. At each DMA-APM voltage 
combination, the CPC reading was averaged for $5 \mathrm{~s}$. Between each APM voltage setting, there was a delay time of $10 \mathrm{~s}$ to ensure system equilibration. After an APM step procedure completed, we increased the DMA voltage by a factor of 1.116 and started a new APM step routine. This process was repeated until the target particle size-mass range was covered. 


\section{Relation between Applied DMA and APM Voltages to Particle Diameter and Mass}

Voltages applied to the DMA and APM need to be converted to particle size and mass to obtain a nominal 2D size-mass spectrum (not accounting for instrument transmission). Since the particles utilized in this study were spherical, the following relationship was utilized to relate DMA voltages to particle diameter:

$$
Z_{p}=\frac{Q \ln \left(\frac{R_{2}}{R_{1}}\right)}{2 \pi L V}=\frac{z e}{3 \pi \mu d_{p}}\left(1+\left(\frac{2 \lambda}{d_{p}}\right)\left\{1.257+0.4 \exp \left[-1.1 /\left(\frac{2 \lambda}{d_{p}}\right)\right]\right\}\right)
$$

where $Z_{p}$ is ion mobility of the particle, $Q$ is the DMA sheath flowrate, $R_{2}$ and $R_{1}$ are the outer and inner DMA radii, $L$ is the length of the classification region, $V$ is the applied DMA voltage, $z$ is the number of elementary charges on the particle, $\mu$ is the carrier gas viscosity, $d_{p}$ is the particle size and $\lambda$ is the mean free path of carrier gas molecules. For the APM, the following equation was used convert applied voltage to particle mass,

$$
m_{p}=\frac{z e V}{\omega^{2} r_{c}^{2} \ln \left(\frac{r_{2}}{r_{1}}\right)}
$$

where $m_{p}$ is particle mass, $\omega$ is the rotational speed of the APM, $r_{c}, r_{1}$ and $r_{2}$ are center, inner and outer radii of the APM, respectively, and $V$ is the applied APM voltage. 


\section{The IM-MS System Transfer Function}

The two dimensional size-mass distribution, $\frac{\partial^{2} n}{\partial d_{p} \partial m_{p}}$ is related to CPC readings via the following equation:

$$
N_{i, j}=\sum_{z=0}^{\infty} \int_{0}^{\infty} \int_{0}^{\infty} T_{i, j}\left(z, d_{p}, m_{p}\right) \frac{\partial^{2} n}{\partial d_{p} \partial m_{p}} d d_{p} d m_{p}
$$

where $N_{i, j}$ is the observed number concentration by the CPC at the $\mathrm{i}^{\text {th }}$ applied DMA voltage and $\mathrm{j}^{\text {th }}$ applied APM voltage, and $z$ is the number of of elementary charges on a particle. $T_{i, j}\left(z, d_{p}, m_{p}\right)$ is the combined transfer function of the entire experimental apparatus for particles with diameter $d_{p}$ and mass $m_{p}$, and $d^{2} n\left(d_{p}, m_{p}\right)$ is the number concentration of particles within the diameter range of $\left(d_{p}, d_{p}+d d_{p}\right)$ and the mass range of $\left(m_{p}, m_{p}+d m_{p}\right) . T_{i, j}\left(z, d_{p}, m_{p}\right)$ can be further expanded as follows,

$$
T_{i, j}\left(z, d_{p}, m_{p}\right)=\Lambda_{i}\left(z, d_{p}\right) \Theta_{j}\left(z, d_{p}, m_{p}\right) \epsilon_{C P C}\left(d_{p}\right) \epsilon_{T}\left(d_{p}\right) f\left(z, d_{p}\right)
$$

where $\Lambda_{i}\left(z, d_{p}\right)$ is the DMA transfer function at the $\mathrm{i}^{\text {th }}$ DMA voltage, ${ }^{4} \Theta_{j}\left(z, d_{p}, m_{p}\right)$ is the APM transfer function at the $\mathrm{j}^{\text {th }}$ APM voltage, ${ }^{5-7} \epsilon_{C P C}\left(d_{p}\right)$ is the CPC detection efficiency, ${ }^{3} \epsilon_{T}\left(d_{p}\right)$ is the transport efficiency through system tubing, ${ }^{8}$ and $f\left(z, d_{p}\right)$ is the fraction of particles exiting the charge conditioner with diameter $d_{p}$ and charge state $z .{ }^{1,9}$ The functional form utilized for each instrument component in equation (S2) can be found in Buckley et $\mathrm{al}^{10}$ and Chen et al. ${ }^{11}$ 


\section{Size-Mass Spectra of Solid Silica Particles}

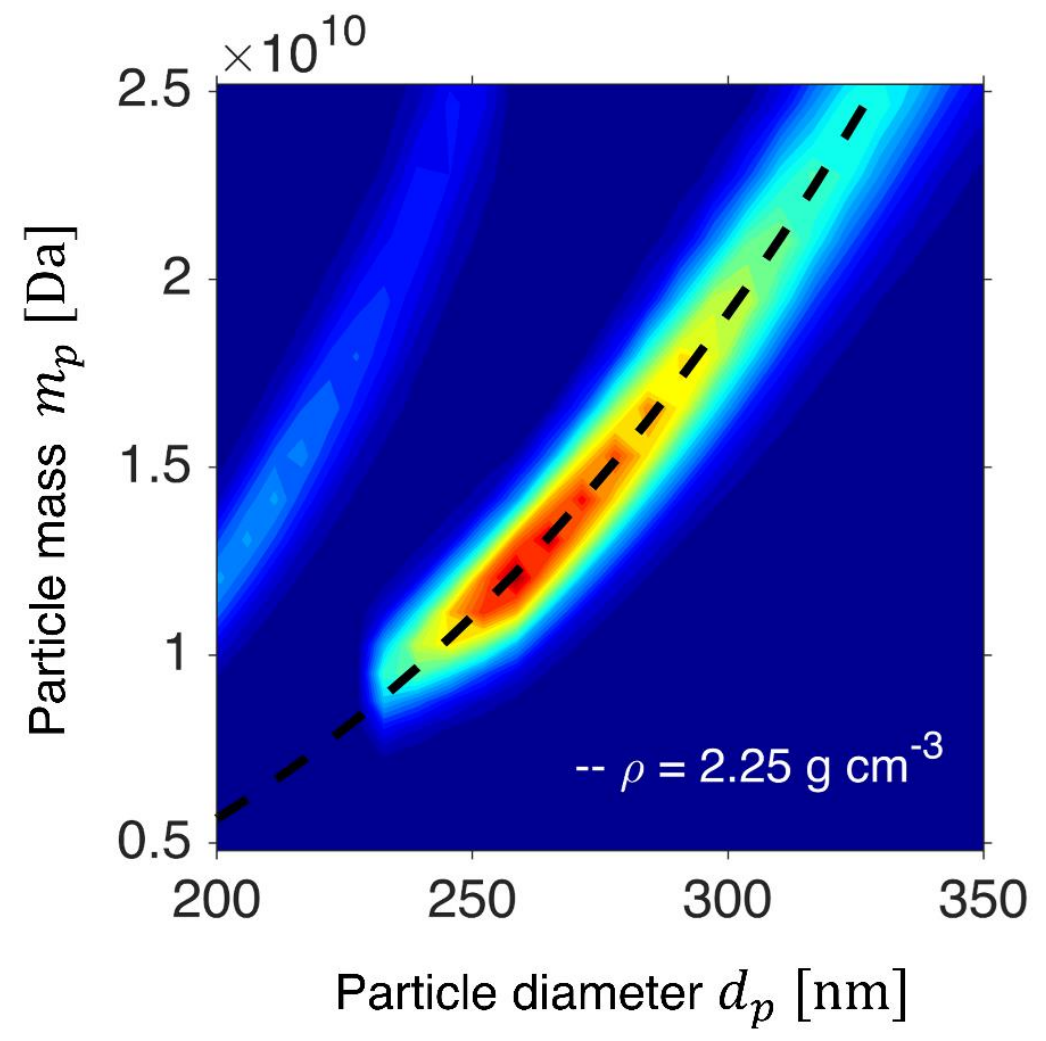

Figure S3. Smoothed 2D size mass-spectra of solid silica particles.

Figure S3 shows a normalized, smoothed size-mass contour plot (uninverted) of solid silica particles synthesis via the Stober process, with a constant density line reprensenting

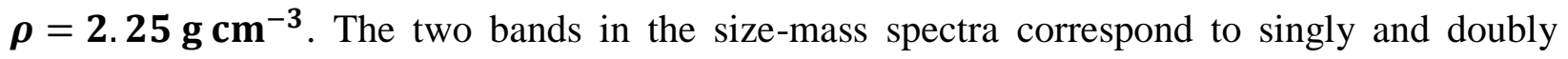
charged particles, respectively. Silica nanoparticles were synthesized by combining $7.09 \mathrm{~mL}$ ethanol, $1.34 \mathrm{~mL}$ TEOS (neat), $1.28 \mathrm{~mL}$ of ultrapure water, and $0.29 \mathrm{~mL}$ of $28-33 \% \mathrm{NH}_{4} \mathrm{OH}$. The solution was allowed to stir for 48 hours at $25{ }^{\circ} \mathrm{C}$. After 48 hours, PEG and chlorotrimethylsilane were added dropwise. The solid silica nanospheres were purified via centrifugation, and then the particles were suspended in 200 proof ethanol. 


\section{TEM Images of Size Selected UMN Particles}

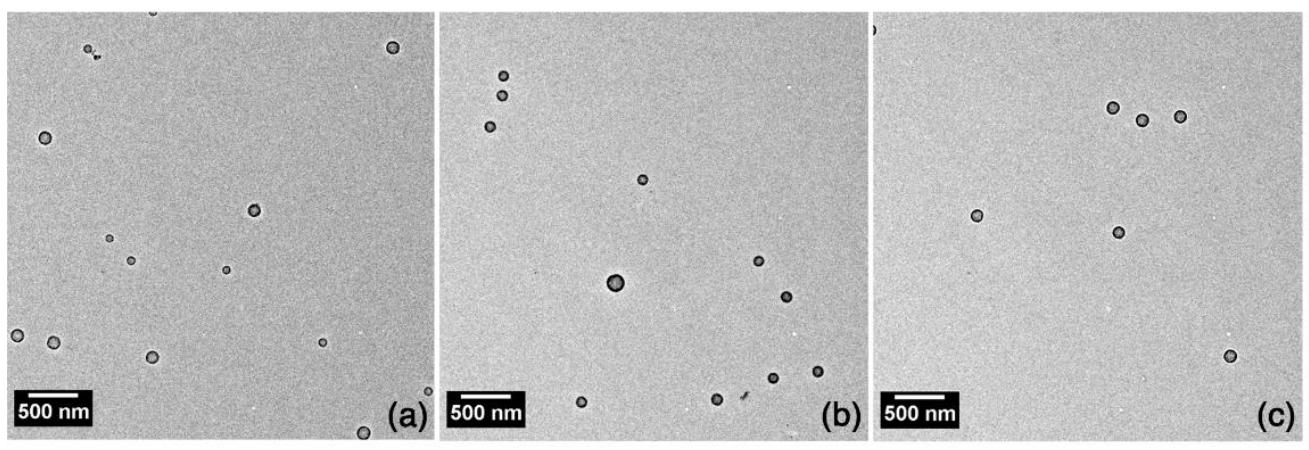

Figure S4. Batch A UMN particles collected at DMA voltages corresponding to (a) $100 \mathrm{~nm}$, (b) $120 \mathrm{~nm}$ and (c) $150 \mathrm{~nm}$ singly charged particles.

It is important to confirm proper operation of the DMA for $2 \mathrm{D}$ data inversion. Figures S4a-c show TEM images of batch A UMNs collected by an aerosol sampler (TSI model 3089) downstream of the DMA, with the DMA voltages set to select $100 \mathrm{~nm}, 120$ $\mathrm{nm}$, and $150 \mathrm{~nm}$ singly charged particles, respectively. Notably in Figure S4a, there are two different sizes of particles; the mean diameter of the small particles is $96.9 \pm 1.9 \mathrm{~nm}$, while the mean diameter of the larger particles is $146.3 \pm 4.4 \mathrm{~nm}$. According to Equation (S3), doubly charged particles with diameters of $~ 151.6 \mathrm{~nm}$ have the same ion mobility as $100 \mathrm{~nm}$ singly charged particles. Therefore, the two particle populations in Figure S4a correspond to singly and doubly charged particles, respectively. In Figure S4b, the singly charged particles have a diameter of $118.5 \pm 5.4 \mathrm{~nm}$, and the doubly charged particles are scarce; in Figure S4c only singly charged particles with a diameter of $139.4 \pm 4.0 \mathrm{~nm}$ were observed. The absence of doubly charged particles in Figure S4c is due to the low concentrations of larger particles in the UMN sample, as doubly charged particles need to be as large as $235.6 \mathrm{~nm}$ in diameter to appear in Figure S4c. Figure 3d in the main text show that particles of this size are very low in number concentration in batch A. 


\section{Replicate Measurements of UMNs}

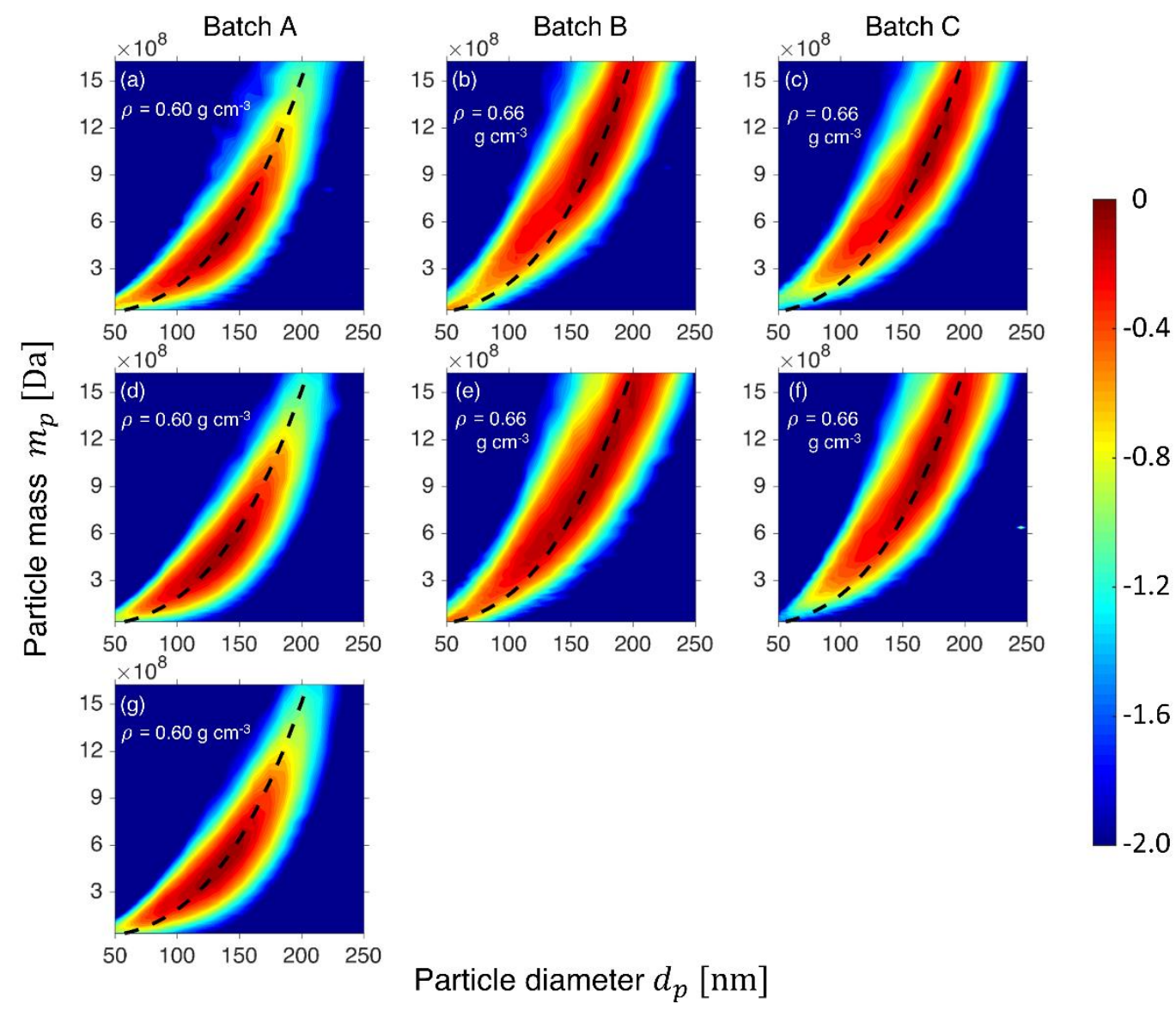

Figure S5. Size-mass spectra of all UMN measurements prior to data inversion. The text above each column of the figures indicates the corresponding UMN batch. Figures S5a-c are the same as Figure 3a-c in the main text and they are shown here again to facilitate comparison. The data shown here are normalized by dividing by the maximum value in the corresponding plot and the color axis corresponds to $\log _{10}$ values of the normalized data. Constant density scaling lines that match with peak concentrations are plotted with densities of $\rho=0.60 \mathrm{~g} \mathrm{~cm}^{-3}, \rho=0.66 \mathrm{~g} \mathrm{~cm}^{-3}$ and $\rho=0.66 \mathrm{~g} \mathrm{~cm}^{-3}$ for UMN batches A, B, and C, respectively.

To ascertain that the observed difference between UMN batches originates from inherent particle properties rather than time variations of instrument settings, replicate measurements for all three batches of UMNs were performed. In total, three measurements were done for batch A (since we were using batch A to compare IM-MS results with TEM results, we put more effort in characterizing this batch), and two measurements each for batch B and C. The measurements were done in a sequence of $A \rightarrow B \rightarrow C \rightarrow A \cdots$ and were finished within two weeks. All the 
normalized size-mass spectra prior to inversion are presented in Figure S5. Figure S5 shows that the 2D spectra for all three batches are highly repeatable. We refer readers to Table 1 in the main text for a quantitative comparison of replicate measurements. 


\section{$\mathbf{N}_{2}$ Physisorption Measurements}

Particle porosity and surface area were measured via nitrogen physisorption on a Micromeritics ASAP $2020 \mathrm{~N}_{2}$ physisorption instrument. UMNs were initially dried under vacuum from ethanol and then further degassed for 5 hours at $120{ }^{\circ} \mathrm{C}$. While degassing, the sample holder was evacuated to $20 \mu$ Torr, at $90{ }^{\circ} \mathrm{C}$, with an evacuation rate of 5 mTorr s${ }^{-1}$. The samples were then heated to $120{ }^{\circ} \mathrm{C}$, with a ramp rate of $10{ }^{\circ} \mathrm{C} \min ^{-1}$; once $120{ }^{\circ} \mathrm{C}$ was reached, the temperature was held constant for 300 minutes. Samples were then analyzed in the ASAP 2020 analysis port under cryogenic temperatures with liquid nitrogen. The surface area and pore size of the particles were determined by the Barrett-Joyner-Halenda (BJH) method.

\section{Mass Correction for a Water Monolayer}

If a water monolayer is present on the pore surfaces of the aerosolized UMNs, the corrected mass of the particle can be calculated with the equation:

$$
m_{p, r}\left(1+\frac{A_{s} \cdot m_{w}}{c s_{w}}\right)=m_{p}
$$

where $\boldsymbol{m}_{\boldsymbol{p}, \boldsymbol{r}}$ is the corrected particle mass, $\boldsymbol{A}_{\boldsymbol{s}}$ is the particle surface area calculated by multiplying particle mass with specific particle surface area determined from the Barrett, Joyner, and Halenda $(\mathrm{BJH})$ method, and $\boldsymbol{c} \boldsymbol{s}_{\boldsymbol{w}}$ is the water molecule cross section calculated by assuming that water molecules are spherical with bulk density. Equation (S5) assumes that all particles have the same specific surface area. 


\section{Comparison of $\frac{d n}{d d_{p}}$ between IM-MS, DLS and TEM}

Number-based particle size distributions $\left(\frac{d n}{d d_{p}}\right)$ for three batches of UMNs were calculated with equation (2) in the main text. The normalized results (that is, the integrated area below the curve is unity) are shown in Figures S6a-c with green curves. Notably, the particle size distributions are bimodal/multimodal, with the first mode of particles not representing UMNs, but instead particles formed by chemical residue during electrospray. To obtain the true UMN size distributions, we fitted two Gaussian distributions for each batch of particles. The blue and black dashed lines in Figures S6a-c show the fit to residue and UMN particles, respectively. To confirm that (a) the first particle mode indeed originates from chemical residues, and (b) the IM-MS-based results reflects the true particle size distribution function, we further analysed TEM images of batch A UMNs. A particle size distribution function based on image analysis of $\sim 400$ particles is shown as a histogram in Figure S6a. Notably, the shape of the TEM-based distribution is very close to the Gaussian fit for the UMNs.

We remark that particles formed by chemical residues influence particle number distributions non-negligibly at small particle sizes. For nanoparticle samples where the mean particle size overlaps with the residue particle size distribution, a careful deconvolution of the true particle size distribution from the residue particles is required (alternatively, the aqueous nanoparticle solutions can be diluted to minimize the residue particle size, but at the cost of signal). For this study, however, the mean UMN particle size $(\geq 100 \mathrm{~nm})$ is well above the residue particle size. This is corroborated by Figure S4a that shows residue particles do not reach a diameter of $100 \mathrm{~nm}$. The main quantities we are concerned with, i.e. mean specific pore volume, are predominantly determined by 
large particles. Therefore, residue particles minimally influence the results presented in this study.

We further compared IM-MS results with dynamic light scattering (DLS). The hydrodynamic diameter and polydispersity index of the UMNs were determined by DLS on a Brookhaven BIC ZetaPals, with a $35 \mathrm{~mW} 600 \mathrm{~nm}$ laser at room temperature. Nanoparticles were first dried from ethanol under vacuum, then suspended in ultrapure water to make $1 \mathrm{mg} \mathrm{mL}^{-1}$ nanoparticle suspensions. The mean particle size and polydispersity values were determined from 3 one-minute measurement runs, with scans at approximately every second. Figure S6d shows mean particle size and polydispersity derived from IM-MS (based on the Gaussian fits shown in Figures S6a-c) and DLS. The DLS-based mean sizes are higher than those inferred from both IM-MS and TEM, consistent with our previous report on UMN sizes. ${ }^{12}$ Figure S6d also shows that DLS reports smaller polydispersity indexes (PDI), but this is mainly because the particle mean diameter is overestimated by DLS, as polydispersity is determined by taking the square of the distribution width over the mean, [width/mean $]^{2}$. A polydispersity index value $\leq$ 0.2 can be considered moderately monodisperse. Figure S6e shows the diameter distribution function of the nanoparticles $\left(G\left(d_{p}\right)\right)$ and signal counts from DLS, and Figure S6f shows the distribution functions using a mathematical transform of raw data in an effort to better fit results for multimodal distributions. We remark that DLS is a light scattering technique that is not capable of making single particle measurements. Therefore, the mode of the data can be greatly skewed by spurious data (Figures S6e-f). ${ }^{13}$ 
(a)

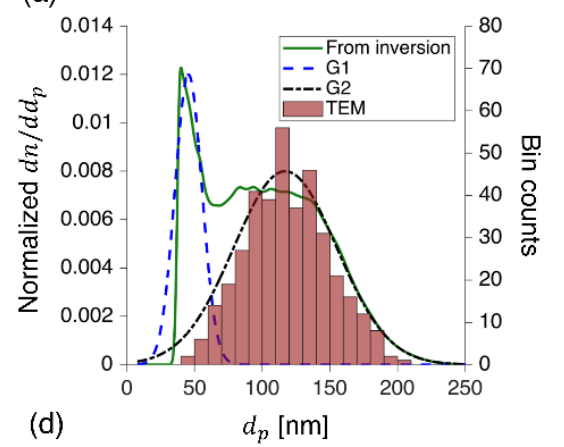

d)

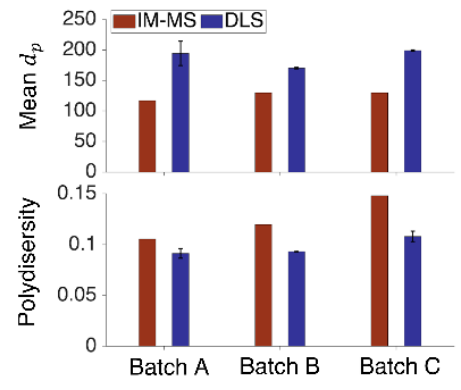

(b)

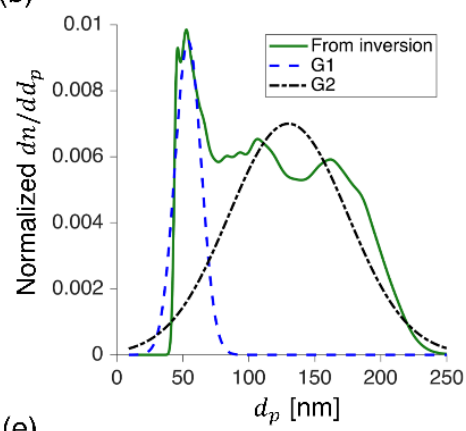

(e)

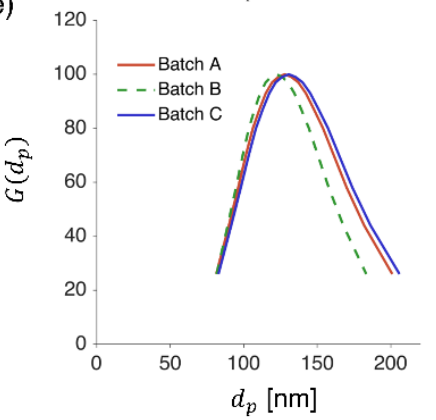

(c)

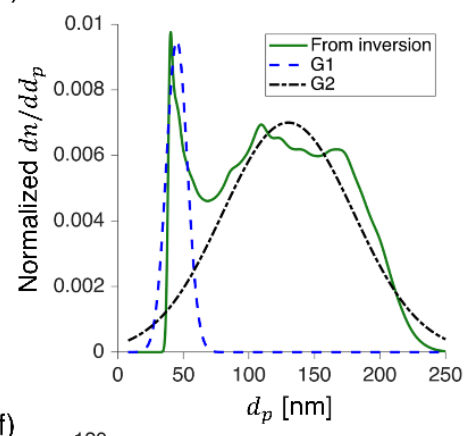

(f)

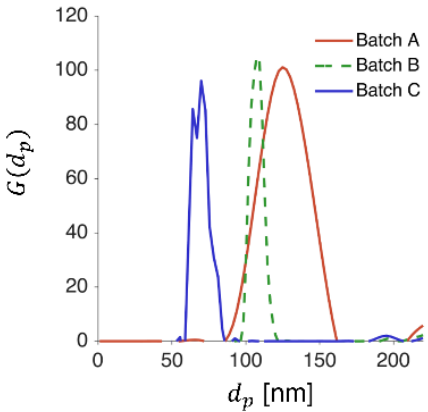

Figure S6. Comparison of IM-MS, DLS and TEM results. (a)-(c) Number-based particle size distributions from IM-MS (green solid lines) and Gaussian fits of different particles modes (blue and black dashed lines). Particles size distribution based on TEM images analysis of batch A UMNs is also shown a histogram in (a). (d) Mean particle diameter and polydispersity index determined by IM-MS and DLS. Polydispersity is determined by taking the square of the distribution width over the mean $\left([\text { width/mean }]^{2}\right)$. (e) Lognormal distribution of the nanoparticles diameter and signal counts showing where maximum amount of signal was located. (f) Multimodal distribution of signal as function of diameter size from DLS measurement. 


\section{References}

1. Wiedensohler, A., An Approximation of the Bipolar Charge-Distribution for Particles in the Sub-Micron Size Range. J. Aerosol Sci 1988, 19 (3), 387-389.

2. Knutson, E. O.; Whitby, K. T., Aerosol Classification By Electric Mobility: Apparatus, Theory, and Applications. J. Aerosol Sci 1975, 6, 443-451.

3. Stolzenburg, M. R.; Mcmurry, P. H., An Ultrafine Aerosol Condensation Nucleus Counter. Aerosol Sci. Technol. 1991, 14 (1), 48-65.

4. Stolzenburg, M. R.; McMurry, P. H., Equations governing single and tandem DMA configurations and a new lognormal approximation to the transfer function. Aerosol Sci. Technol. 2008, 42 (6), 421-432.

5. Olfert, J. S., A numerical calculation of the transfer function of the fluted centrifugal particle mass analyzer. Aerosol Sci. Technol. 2005, 39 (10), 1002-1009.

6. Tajima, N.; Sakurai, H.; Fukushima, N.; Ehara, K., Design Considerations and Performance Evaluation of a Compact Aerosol Particle Mass Analyzer. Aerosol Sci. Technol. 2013, 47 (10), 1152-1162.

7. Lin, G. Y.; Liao, B. X.; Tzeng, N. J.; Chen, C. W.; Uang, S. N.; Chen, S. C.; Pui, D. Y. H.; Tsai, C. J., The Effect of Nanoparticle Convection-Diffusion Loss on the Transfer Function of an Aerosol Particle Mass Analyzer. Aerosol Sci. Technol. 2014, 48 (6), 583-592.

8. Gormley, P. G.; Kennedy, M., Diffusion from a stream flowing through a cylindrical tube. Proc. Irish Royal Academy 1949, 52A, 163-169.

9. Gopalakrishnan, R.; Meredith, M. R.; Larriba-Andaluz, C.; Hogan, C. J., Brownian Dynamics Determination of the Bipolar Steady State Charge Distribution on Spheres and Nonspheres in the Transition Regime. J. Aerosol Sci 2013, 63, 126-145.

10. Buckley, D. T.; Kimoto, S.; Lee, M.-H.; Fukushima, N.; Hogan, C. J., Technical note: A corrected two dimensional data inversion routine for tandem mobility-mass measurements. $J$. Aerosol Sci 2017, 114, 157-168.

11. Chen, X.; Ghosh, S.; Buckley, D. T.; Mohan Sankaran, R.; Hogan, C. J., Characterization of the state of nanoparticle aggregation in non-equilibrium plasma synthesis systems. J. Phys. D: Appl. Phys. 2018, 51 (33), 335203.

12. Lee, A. L.; Gee, C. T.; Weegman, B. P.; Einstein, S. A.; Juelfs, A. R.; Ring, H. L.; Hurley, K. R.; Egger, S. M.; Swindlehurst, G.; Garwood, M.; Pomerantz, W. C. K.; Haynes, C. L., Oxygen Sensing with Perfluorocarbon-Loaded Ultraporous Mesostructured Silica Nanoparticles. ACS Nano 2017, 11 (6), 5623-5632.

13. Anderson, W.; Kozak, D.; Coleman, V. A.; Jämting, Å. K.; Trau, M., A comparative study of submicron particle sizing platforms: Accuracy, precision and resolution analysis of polydisperse particle size distributions. J. Colloid Interface Sci. 2013, 405, 322-330. 